\title{
Magnetic fields in AGB stars and (proto-) Planetary Nebulae
}

\author{
Fabrice Herpin ${ }^{1,2}$, A. Baudy ${ }^{1,2}$, E. Josselin ${ }^{3}$, C. Thum ${ }^{4}$ \\ and $\mathrm{H}$. Wiesemeyer ${ }^{4}$ \\ ${ }^{1}$ Université de Bordeaux, Lab. d'Astrophysique de Bordeaux, F-33000 Bordeaux, France \\ email: herpin@obs.u-bordeaux1.fr \\ ${ }^{2}$ CNRS/INSU, UMR 5804, BP 89, 33271 FLOIRAC cedex, France \\ ${ }^{3}$ GRAAL, Université Montpellier II - ISTEEM, CNRS, Place Eugène Bataillon, F-34095 \\ Montpellier Cedex, France \\ ${ }^{4}$ Institut de Radio Astronomie Millimétrique, 300 rue de la Piscine, F-38406 Saint Martin \\ d'Hères, France
}

\begin{abstract}
During its quick transition to the Planetary Nebula stage, the Asymptotic Giant Branch star will completely change its geometry. This AGB stellar evolution stage is characterized by a high mass loss driven by the radiation pressure. Strong magnetic field may rule the mass loss geometry and the global shaping of these objects. Following our previous work on the polarization of the $\mathrm{SiO}$ maser emission in a representative sample of O-rich evolved stars, we present here a study towards C-rich objects and PPN/PN objects to obtain unbiased conclusions. Using Xpol at the IRAM-30 m telescope, we have conducted CN N=1-0 observations to investigate the Zeeman effect in this molecule and draw conclusion on the evolution of the magnetic field and its influence during the transition of an AGB star to the PN stage. Following the analysis described by Crutcher et al. (1996) we derive an estimate of the magnetic field.
\end{abstract}

Keywords. Magnetic field - stars: evolution - radio lines: stars - ISM: molecules

\section{Introduction}

The prodigious mass loss observed in the numerous and widespread evolved stars make these objects the main recycling agents of the interstellar medium, and thus one of the most important objects in the Universe. During its quick transition to the Planetary Nebula (hereafter PN) stage, the Asymptotic Giant Branch (hereafter AGB) star will completely change its geometry: the quasi-spherical object becomes axisymmetrical, point symmetrical or even shows more high-order symmetries (e.g. Sahai \& Trauger 1998). The classical or generalized Interacting Stellar Winds models (cf. Kwok 2000) try to explain this shaping, but have serious difficulties in producing complicated structures with peculiar jets or ansae and do not fully address the origin of the wind.

Strong magnetic field may rule the mass loss geometry and could thus determine the global shaping of these objects. Some recent studies tend to demonstrate the importance of magnetic field in evolved objects. Bujarrabal et al. (2001) show that in $80 \%$ of the PPNe from their sample the fast molecular flows have too high momenta to be powered by radiation pressure (1000 times larger in some cases) what may be explained by magnetic field. Recently, magnetic field was discovered for the first time in central stars of PN (Jordan et al. 2005) and estimated to be at the kiloGauss level, but polarimetric observations toward AGB stars are needed to constrain the magnetic field strength. Moreover, new models involving the magnetic field B were developed where B plays the role of a catalyst and of a collimating agent. 


\section{Magnetic field in AGB stars}

The circumstellar envelope of evolved stars can be probed at different depths through the study of the maser emission of three different molecules, $\mathrm{OH}, \mathrm{H}_{2} \mathrm{O}$ and $\mathrm{SiO}$, located at different distances from the central star, respectively at 1000-10000, a few 100 and 5-10 AU (one stellar radius $\mathrm{R}_{\star} \sim 1 \mathrm{AU}$ ).

Measurement of the $\mathrm{SiO}$ maser radiation polarization can lead to an estimation of the value of magnetic field, $B_{/ /}$on the line of sight (for a single dish antenna), or can reveal the structure of the magnetic field (interferometric observations). Until now numerous polarimetric observations of $\mathrm{OH}$ masers have been done, several of $\mathrm{H}_{2} \mathrm{O}$ masers, but few of $\mathrm{SiO}$ maser emission. It must be stressed that $\mathrm{SiO}$ is a non-paramagnetic species. The Zeeman splitting exists but the sublevels overlap; the effect is thus undetectable and hence only net polarization can be used to trace magnetic fields. The current state of the knowledge of $\mathrm{B}$ is:

- between 1000-10000 AU, B// 5-20 mG (OH masers, e.g. Kemball \& Diamond 1997, Szymczak \& Cohen 1997),

- at a few $100 \mathrm{AU}$ from the star, $B_{/ /} \sim$ a few $100 \mathrm{mG}\left(\mathrm{H}_{2} \mathrm{O}\right.$ masers, e.g. Vlemmings, Diamond \& van Langevelde 2001),

- at 5-10 AU, B// $\sim 5-10 \mathrm{G}$ (SiO masers; Kemball \& Diamond 1997, VLBI observations in TX Cam).

\section{The $\mathrm{SiO}$ maser polarization results}

Simultaneous spectroscopic measurement of the 4 Stokes parameters (cf. Fig. 1) were carried out towards 57 O-rich evolved stars by Herpin et al. (2006) via observations of the $\mathrm{SiO}(\mathrm{v}=1, \mathrm{~J}=2-1)$ line at $86.243 \mathrm{GHz}$. These observations were performed with the IF polarimeter installed at the IRAM 30m telescope at Pico Veleta, Spain (Thum et al. 2003). From the Stokes parameters measurements one deduces for each velocity channel:

- the circular polarization rate $p_{C}=V / I$

- the linear polarization rate $p_{L}=\sqrt{Q^{2}+U^{2}} / I$

- the polarization angle $\chi=\frac{\arctan (U / Q)}{2}$

Assuming Elitzur et al. (1996) maser theory, we calculate the mean value of the magnetic field $B_{/ /}$for each $\mathrm{SiO}$ maser component (cf. Herpin et al. 2006). B// is between 0 and 18 Gauss, with a mean value of $3.5 \mathrm{G}$. This value combined with the strength of the field in more outer layers of the envelope (given by $\mathrm{OH}$ and $\mathrm{H}_{2} \mathrm{O}$ masers) agrees with a variation law for $\mathrm{B}$ in $1 / r$.

The main bias in this study was the source sample, because $\mathrm{SiO}$ maser emission is only present in O-rich evolved objects and disappears soon after the star has reached the end of the AGB (Nyman et al. 1998), this method cannot be used in C-rich objects or PPN/PN. Moreover, no $\mathrm{OH} / \mathrm{H}_{2} \mathrm{O}$ maser lines are detected toward C-rich stars (e.g., Szczerba et al. 2002). As a consequence, none of the maser molecules can be used to estimate the magnetic field in these objects. Nevertheless, the same type of study we did in O-rich stars should also be conducted within C-rich objects to obtain unbiased conclusions on AGB stars and to investigate the evolution of the magnetic field and its influence during the transition of an AGB star to the PN stage.

\section{CN as a tracer of the field in carbon stars ?}

$\mathrm{CN}$ seems to be a good molecular tracer to perform such studies in carbon stars. First of all, the $\mathrm{N}=1 \rightarrow 0$ and $\mathrm{N}=2 \rightarrow 1$ lines have already been observed and easily detected 

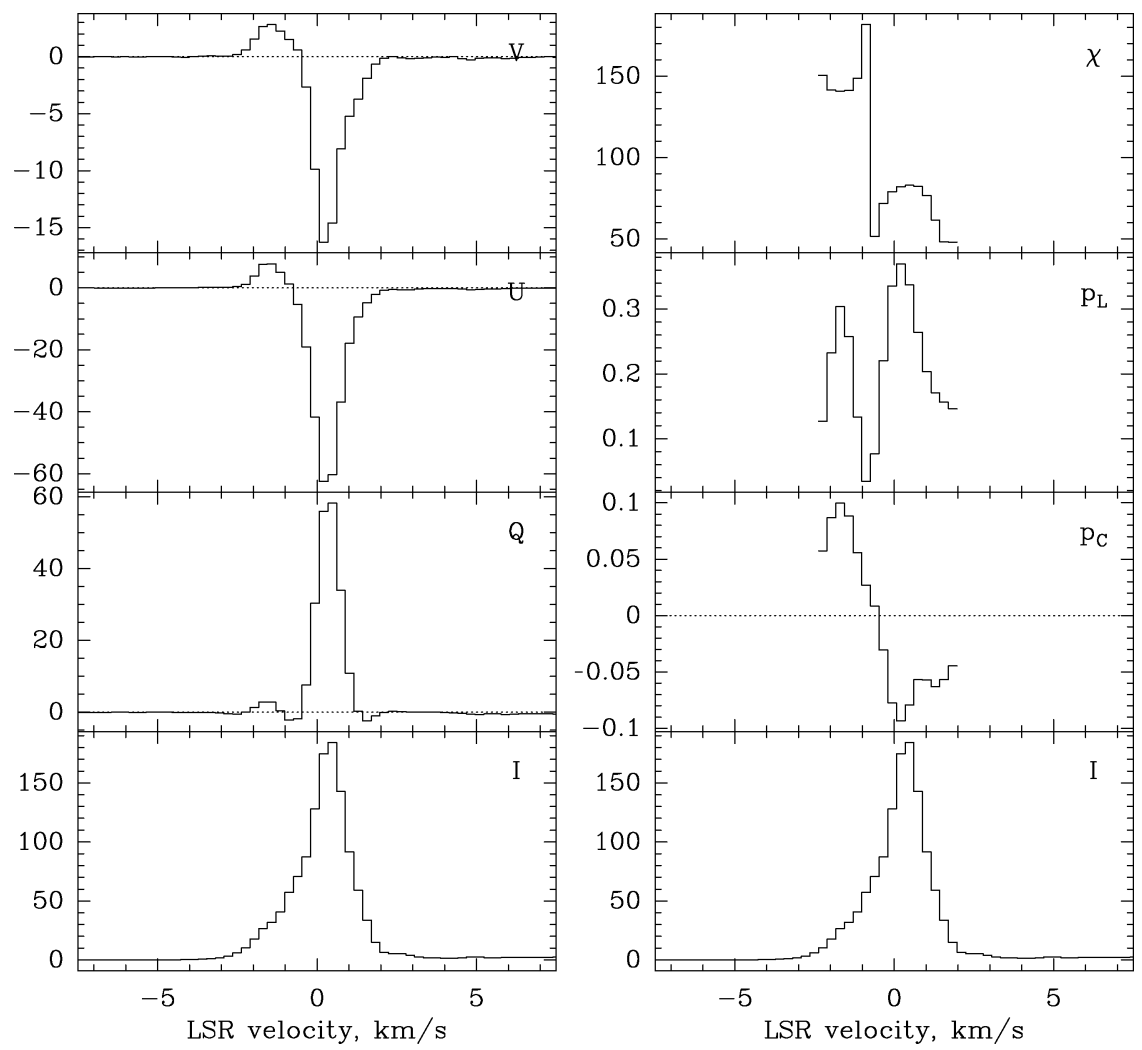

Figure 1. Left: V, U, Q et I Stokes parameters for R Leo (given in Kelvins $T_{m b}$; SiO maser observations). Right: derived position angle of polarization $(\chi)$ in degrees, linear $\left(p_{L}\right)$ and circular $\left(p_{C}\right)$ polarization levels and intensity for R Leo.

Table 1. Zeeman Splittings for $\mathrm{CN} \mathrm{N}=1 \rightarrow 0$ (Crutcher et al. 1996). R.I. stands for Relative Intensity in LTE conditions.

\begin{tabular}{|c|c|c|c|c|}
\hline$\left(\mathbf{N}^{\prime}, \mathbf{J}^{\prime}, \mathbf{F}^{\prime}\right) \rightarrow(\mathbf{N}, \mathbf{J}, \mathbf{F})$ & $\nu_{0}(\mathrm{GHz})$ & $\mathrm{Z}\left(\mathrm{Hz} \mu \mathrm{G}^{-1}\right)$ & R.I. & $\mathrm{Z} \times$ R.I. \\
\hline 1. $(1,1 / 2,1 / 2)-$ & 113.14434 & 2.18 & 8 & 17.4 \\
\hline 2. $(1,1 / 2,3 / 2) \rightarrow(0,1 / 2,1 / 2)$ & 113.17087 & -0.31 & 8 & 2.5 \\
\hline 3. $(1,1 / 2,3 / 2) \rightarrow(0,1 / 2,3 / 2)$ & 113.19133 & 0.62 & 10 & 6.2 \\
\hline 4. $(1,3 / 2,3 / 2) \rightarrow(0,1 / 2,1 / 2)$ & 113.48839 & 2.18 & 10 & 21.8 \\
\hline 5. $(1,3 / 2,5 / 2) \rightarrow(0,1 / 2,3 / 2)$ & 113.49115 & 0.56 & 27 & 15.1 \\
\hline 6. $(1,3 / 2,1 / 2) \rightarrow(0,1 / 2,1 / 2)$ & 113.49972 & 0.62 & 8 & 5.0 \\
\hline 7. $(1,3 / 2,3 / 2) \rightarrow(0,1 / 2,3 / 2)$ & 113.50906 & 1.62 & 8 & 13.0 \\
\hline
\end{tabular}

at the 30m by Bachiller et al. (1997a,b) and Josselin \& Bachiller (2003) towards these objects. Moreover, $\mathrm{CN}$ is a paramagnetic species, thus exhibiting Zeeman splitting in its $3 \mathrm{~mm} \mathrm{~N}=1-0$ line emission when the spectral line-forming region is permeated by a field $B$. The CN Zeeman signal is thermal (as opposed to $\mathrm{SiO}$ ) and therefore free of the idiosyncrases of maser theory.

CN N=1-0 line has a total of 9 hyperfine components (splitted in two groups, one around $113.17 \mathrm{GHz}$, the other around $113.49 \mathrm{GHz}$ ), with 7 main lines. Of those 7,4 exhibit strong Zeeman effect (see Table 1, line 1,4, 5 and 7). Crutcher et al. (1996) developed an analysis procedure consisting of a least-squares fit in frequency to all seven 

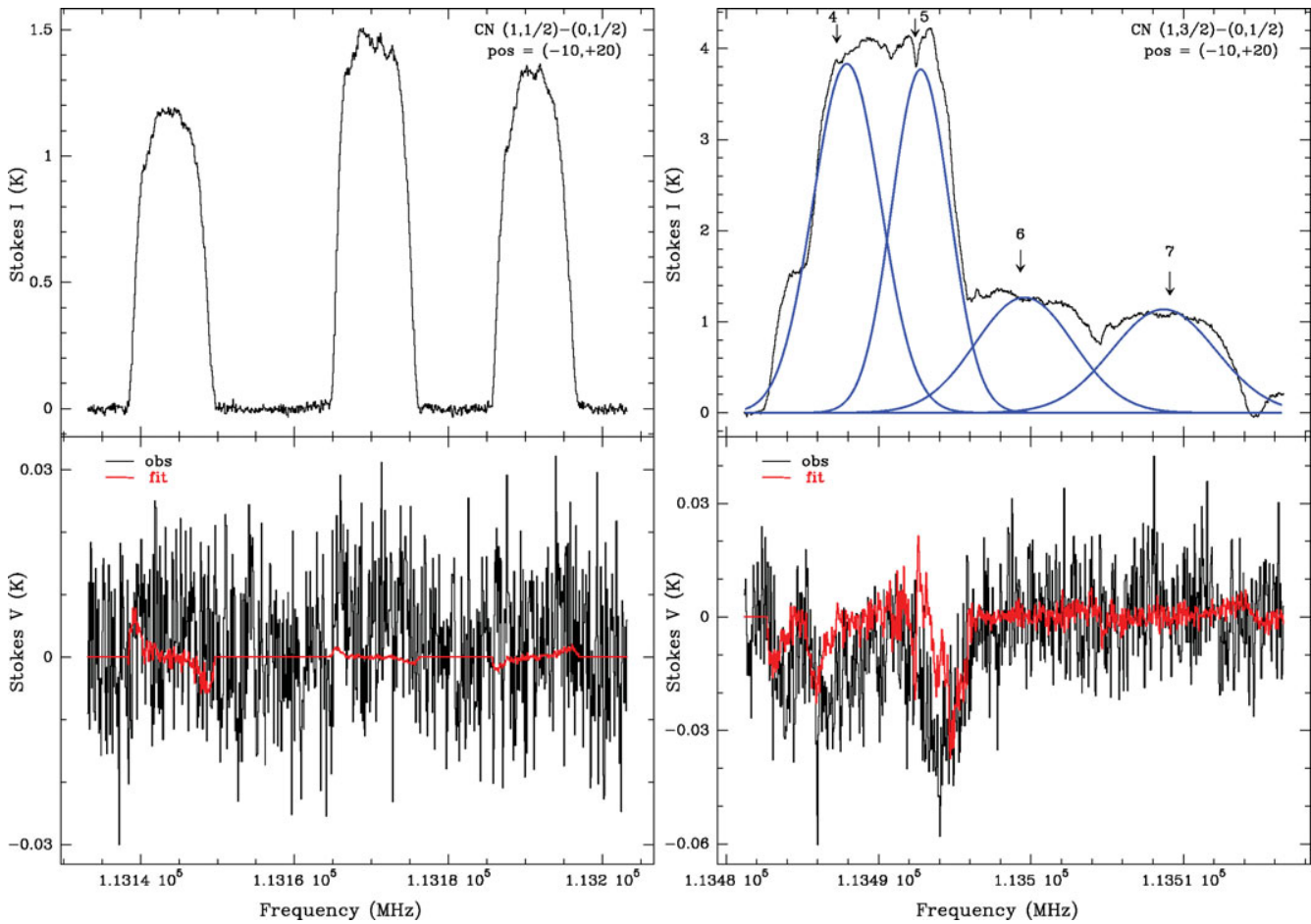

Figure 2. Left: CN $(1,1 / 2) \rightarrow(0,1 / 2)$ Stokes I (Top) and V (Bottom) spectra for IRC +10216 (position $-10 ",+20$ "); Right: same for the CN $(1,3 / 2) \rightarrow(0,1 / 2)$. Observations, gaussian fits for I and least-squares fits for $\mathrm{V}$ are respectively in black, blue and red. No Stokes $\mathrm{V}$ signal is detected toward the non(low)-paramagnetic hyperfines, demonstrating that the detected features are indeed due to Zeeman.

hyperfine transitions of the line $\mathrm{V}$ spectra, making possible to separate the spurious and Zeeman signals.

The main purpose of this work was to measure and analyze the polarization of the CN $\mathrm{N}=1-0$ line emission in a sample of evolved stars: two C-rich AGB stars (RW LMi and IRC +10216 at a distance of respectively 440 and 120 pc), one PPN (AFGL618, at 900 pc), and one $\mathrm{PN}$ object (NGC7027, at $880 \mathrm{pc}$ ). As the diameter of the $\mathrm{CN}$ ring around IRC +10216 is larger than the $30 \mathrm{~m}$ beam, our observations were focused on two selected positions for that source.

Simultaneous spectroscopic measurement of the 4 Stokes parameters for the seven hyperfine transitions given in Table 1 were made in november 2006 with the XPol polarimeter (Thum et al. 2008) at the IRAM-30m. Following the analysis procedure described by Crutcher et al. (1996), we do a least-squares fit in frequency simultaneously to all 7 hyperfine line $\mathrm{V}$ spectra $(\mathrm{i}=1,7)$ :

$$
V_{i}(\nu)=C_{1} I_{i}(\nu)+C_{2}\left(d I_{i}(\nu) / d \nu\right)+C_{3} Z_{i}\left(d I_{i}(\nu) / d \nu\right)
$$

This method enables us to distinguish between the Zeeman effect $\left(C_{3}=B_{/ /} / 2\right)$ and instrumental effects $\left(C_{1,2}\right)$.

\section{CN results and consequences}

From the fit of the observed Stokes V spectra (see Fig. 2 for IRC+10216), we then estimate the magnetic field strength along the light-of-sight within the CN layer for the 
Table 2. For each object in our sample are given for the CN layer the molecular abundance ( $\chi$, relative to $\mathrm{H}_{2}$ ), its distance to the central star in $\mathrm{AU}$ (and in its size in arcseconds). $\mathrm{B}_{8 r_{\star}}$ and $\mathrm{B}_{r_{\star}}$ are the extrapolated strengths of the magnetic field (following a $1 / r$ law) at $8 \mathrm{r}_{\star}$ (equivalent to the $\mathrm{SiO}$ maser zone for O-rich objects) and at one stellar radius.

\begin{tabular}{|c|c|c|c|c|c|}
\hline Object & $\chi(\mathrm{CN})$ & $\begin{array}{l}\mathrm{d}_{C N} \\
{[\mathrm{AU}]}\end{array}$ & $\begin{array}{c}\mathrm{B}_{8 r_{\star}} \\
{[\text { Gauss] }}\end{array}$ & $\begin{array}{c}\mathrm{B}_{r_{\star}} \\
{[\text { Gauss }]}\end{array}$ & $\begin{array}{c}r_{\star} \\
{[\mathrm{AU}]}\end{array}$ \\
\hline & & $2200(5$ & 2 & 10 & 1 \\
\hline IRC & 6.21 & $2500(21 ")$ & 2.7 & 21.6 & 1 \\
\hline $\mathrm{CR}$ & 2.11 & $5000(5 ")$ & $<300$ & $<2400$ & $2.310^{-3}$ \\
\hline NGC7027 & $2.310^{-7}$ & $10000\left(11^{\prime \prime}\right)$ & $<250$ & $<2000$ & $2.310^{-3}$ \\
\hline
\end{tabular}

4 objects: 7.2 (poor S/N ratio) and 8.6 Gauss, respectively for RW LMI and IRC+10216. Only upper limits are derived for CRL618 $(\leqslant 0.9$ Gauss) and NGC7027 $(\leqslant 0.3$ Gauss) as no Stokes V signal is detected.

The objects from our sample have been already observed in CN lines by some authors, making hence possible to estimate the size (and distance to the central object) of the CN layer (see Table 2) that we are investigating through our polarization observations: Lindqvist et al. (2000), Lucas et al. (1995), Josselin et al. (private communication) respectively for RW LMi, IRC+10216 and NGC7027. Only HCN observations are available for CRL618, but HCN being a molecule of photospheric origin that gets photodissociated by the ambient UV-field into CN (e.g. Huggins \& Glassgold 1982), we can assume CN molecules are surrounding the $\mathrm{HCN}$ envelope, leading to a rough estimate of the $\mathrm{CN}$ envelope size.

First of all, the detection or non-detection of the Stoked V signal cannot be explained by the CN abundances as shown in Table 2. Therefore, we checked any instrumental effect or bias that could have lead to a non-detection, but the instrumental polarization is very low and the same for all sources. Furthermore, the size of the CN layer is smaller than the $30 \mathrm{~m}$ beam ( $21^{\prime \prime}$ at that frequency), and the side-lobe contamination has been well constrained.

We also tried to verify that our magnetic field estimates are consistent with previous works. Hence, following Herpin et al. (2006) conclusion, we have applied a $1 / r$ variation law for the magnetic field strength across the envelope. We have therefore extrapolated the $\mathrm{B}$ value at a distance of 8 stellar radius, roughly the same distance than the $\mathrm{SiO}$ layer in O-rich objects and at one stellar radius. For AGB objects, the derived strength of the magnetic field is between 2 and 2.6 Gauss, entirely compatible with the estimate of $\mathrm{B}$ by Herpin et al. (2006) in O-rich objects from SiO maser observations. In PPN and PN objects, we have estimated upper limits for B of 2000 and 2400 kGauss at 1 $r_{\star}$ respectively in CRL618 and NGC7027, in agreement with Jordan et al. (2005), who measured magnetic field of a few kGauss at that distance to the central star.

We then conclude that our CN results are completely reliable and consistent. Moreover, even for AGB stars, the strength of the magnetic field at the stellar surface is strong enough to allow B to play a major role in the object shaping.

\section{Conclusion}

We have extended the magnetic field study in evolved stars made by Herpin et al. (2006) in order to study its evolution and its influence during the transition of an AGB star to the PN stage. We have observed the Zeeman effect in $\mathrm{CN}$ line emission in C-rich objects and more evolved stars (one PPN and one PN). Our B estimates are consistent with 
previous studies and confirm that the magnetic field varies as $1 / r$ accross the circumstellar envelope.

The magnetic field is sufficiently strong to be determinant during the evolution of the AGB star: magnetic field seems to be able to help in the process of collimating, and more generally, of shaping in AGB objects, but is not the main agent at this stage. The increasing strength of the magnetic field in the following stages of evolution, as shown by our results and Jordan et al. (2005), will make B a determinant shaping agent.

\section{References}

Bachiller, R. et al. 1997a, A\&A 319, 235

Bachiller, R. et al. 1997b, A\&\&A 324, 1123

Bujarrabal, V., Castro-Carrizo, A., Alcolea, J., \& Sánchez Contreras, C. 2001, A $\& A$ 377, 868

Crutcher, R. M. et al. 1996, ApJ 456, 217

Elitzur, M. 1996, ApJ 457, 415

Herpin, F., Baudry, A., Thum, C., Morris, D., \& Wiesemeyer, H. 2006, A $\& A$ 450, 667

Huggins, P. J. \& Glassgold, A. E. 1982, AJ 87, 1828

Jordan, S., Werner K., \& O'Toole, S. J. 2005, A\&A 432, 273

Josselin, E. \& Bachiller, R. 2003, A\&SA 397, 659

Kemball, A. J. \& Diamond, P. J. 1997 ApJ 481, L111

Kwok, S. 2000, in The origin and Evolution of Planetary Nebulae, Cambridge Astrophysics Series 31, Cambridge University Press edition

Lindqvist, M. et al. 2000, A\&SA 361, 1036

Lucas, R. et al. 1995, Ap\&SSS 224, 293

Nyman, L.-A., Hall, P. J., \& Olofsson, H. 1998, A\&ASS 127, 185-200

Sahai, R. \& Trauger, J. T. 1998, AJ 116, 1357

Soker, N. 2002, MNRAS 337, 1038

Szczerba, R. et al. 2002, A\&A 381, 491

Szymczak, M. \& Cohen, R. J. 1997, MNRAS 288, 945

Thum, C., Wiesemeyer, H., Morris, D., Navarro, S., \& Torres, M. 2003 SPIE 4843, 272

Thum, C. et al. 2008, PASP 120, 777

Vlemmings, W., Diamond, P. J., \& van Langevelde, H. J. 2001, A\&̛A 375, L1

\section{Discussion}

JORDAN: Did you see any correlation between magnetic field strength and object's asymmetry? If not, this will indicate that the magnetic field does not play any role in the object's shaping.

HERPIN: Actually, the shape of the AGB stars is only known for few objects. It is therefore difficult to conclude on any correlation. Moreover, the shaping occurs - or more exactly is seen - after the star has left the AGB phase.

BECKMAN: How accurate/reliable are the magnetic field strength estimates you make?

HERPIN: For SiO masers, the magnetic field estimate is indeed model-dependent, but the values we derived, assuming Elitzur's theory, are consistent with former water and $\mathrm{OH}$ observations, i.e. $\mathbf{B}$ varying in $1 / r$ across the envelope. The location of $\mathrm{SiO}$ maser cells is also very well known. The Zeeman effect observed for the CN line emission is well understood too. 